\title{
Fluctuational transitions across different kinds of fractal basin boundaries
}

\author{
A. N. Silchenko, S. Beri, D. G. Luchinsky, and P. V. E. McClintock \\ Department of Physics, Lancaster University, Lancaster LA1 4YB, United Kingdom \\ (Received 26 March 2004; revised manuscript received 29 November 2004; published 4 April 2005)
}

\begin{abstract}
We study fluctuational transitions in discrete and continuous dynamical systems that have two coexisting attractors in phase space, separated by a fractal basin boundary which may be either locally disconnected or locally connected. Theoretical and numerical evidence is given to show that, in each case, the transition occurs via a unique accessible point on the boundary, both in discrete systems and in flows. The complicated structure of the escape paths inside the locally disconnected fractal basin boundary is determined by a hierarchy of homoclinic points. The interrelation between the mechanism of transitions and the hierarchy is illustrated by consideration of fluctuational transitions in dynamical systems demonstrating "fractal-fractal" basin boundary metamorphosis at some value of a control parameter. The most probable escape path from an attractor, which can be either regular or chaotic, is found for each type of boundary using both statistical analysis of fluctuational trajectories and the Hamiltonian theory of fluctuations.
\end{abstract}

DOI: 10.1103/PhysRevE.71.046203 PACS number(s): 05.45.Gg, 02.50. - r, 05.20.-y, 05.40.-a

\section{INTRODUCTION}

Examples of fluctuation-induced transitions can be found in many branches of science, from diffusion in solids and protein folding, to switching in lasers [1] and resonantly driven trapped electrons [2]. In many situations, it is important not only to estimate the escape probability, but also to be able to control it, which, in turn, requires additional information about the structure of the escape paths connecting metastable states.

The complex structure of chaotic limit sets existing in phase space, and the fractality of the basin boundaries separating coexisting attractors [3-6], inevitably make the analysis of fluctuational transitions an extremely difficult and challenging problem. The main difficulty stems from delicate questions about the uniqueness of solutions and the boundary condition on the chaotic attractor and/or on the fractal basin boundary (FBB). A number of interesting analytic results were obtained for the escape problem in one- and twodimensional linear chaotic maps [7-9] using the methods of transition state theory. However, exact analytic results are not readily available for the general case of a nonlinear map. Instead, an asymptotic analysis of the escape problem in the limit of weak noise developed for continuous systems (see, e.g., [10]) and extended to maps $[11,12]$ can be adopted for chaotic systems [13-16].

In this approach, an auxiliary Hamiltonian system (for flows), or an auxiliary area-preserving map, is introduced describing the fluctuational motion as a motion along certain deterministic trajectories. The solution of the escape problem is then given by the solution of the boundary value problem in the extended phase space of the auxiliary system: it is the solution yielding the trajectory of minimal energy connecting the attractor to the boundary of its basin of attraction. We emphasise that these deterministic trajectories underlying fluctuational motion are not mere theoretical abstractions, but can be observed experimentally $[17,18]$.

The main difficulty in application of these results to chaotic systems lies in the fact that the boundary conditions are in general not known, neither at the chaotic attractor nor on the fractal basin boundary. For one-dimensional maps, the problem of specifying boundary conditions can sometimes be sidestepped [14] by carrying out minimization over all possible initial conditions on the chaotic attractor. Clearly, such an approach cannot be extended to higher-dimensional fractal structures.

Recently, however, it was shown that the problem of escape in chaotic systems with fractal boundaries can be considerably simplified. First, it was demonstrated [19-21] and independently confirmed [22] that unique boundary conditions can be identified on a chaotic attractor. Second, our recent studies have shown that fluctuational transitions across a locally disconnected (LD) FBB occur via a deterministic mechanism. We have also shown that escape occurs via a unique accessible boundary point on the LD FBB and that the structure of escape paths inside the LD FBB is determined by a hierarchy of points in a homoclinic tangle [23]. Moreover, it was inferred that the mechanism in question should be common among many chaotic maps and flows.

In this paper, we describe in more detail the mechanism of fluctuational transition across both LD and locally connected (LC) FBBs, and we demonstrate that our prediction about the common nature of the described mechanism is indeed correct.

It was shown earlier that the presence of the homoclinic tangencies that cause fractalization of the basin boundary also leads to a decrease in activation energy [24,25]. In the present paper, we give theoretical and numerical evidence to demonstrate that the predicted mechanism is valid for systems showing "fractal-fractal" basin boundary metamorphoses. This allows one to study the activation energy and the optimal transition path even when the fractal boundary and the escape path experience discontinuous changes. We show that the noise can effectively move the accessible point inside the open neighborhood containing an attractor. Finally, by studying fluctuational transitions across an LC FBB, we have been able to find a unique optimal escape path and an optimal fluctuational force, even for this qualitatively different case. 
We emphasize that, by exploitation of an analogy between the variational formulation of the control problem, and noiseinduced escape from the domain of attraction [26], we are enabled to find both the optimal escape path and the corresponding optimal force that causes switching of complex chaotic systems between coexisting stable states. This nonlinear approach to the problem of steered transitions between coexisting stable states is of broad interdisciplinary interest and is closely related to problems of control and stability of complex nonlinear dynamical systems [27,28].

The paper is organized as follows. In Secs. II A and II B, we review briefly some of our relevant recent results, describing in detail the procedures for computation of the activation energy. In Sec. II C, a proof based on topological arguments is given to account for our observation that escape takes place at an accessible point on the basin boundary, and the procedures used for minimization of the activation energy are presented. Section III is devoted to the study of noise-induced escape from a periodic attractor in the Henon map. It is known that, for some values of the control parameter, this system possesses a so-called "fractal-fractal" basin boundary crisis, where the FBB drastically change its form, spreading inside the open neighborhood containing the attractor [29]. We analyze the structure of the optimal escape paths and generalize the notion of a noisy precursor of bifurcation to the interesting case of boundary crisis in the presence of noise. Section IV deals with fluctuation transitions across a closed nowhere-differentiable LC FBB in a nonanalytic quadratic map. Our conclusions are given in Sec. V.

\section{FLUCTUATIONAL TRANSITIONS ACROSS THE LD FBB}

To study fluctuation transitions through an LD FBB, we took as a model the Holmes map [30] driven by noise,

$$
\begin{gathered}
x_{n+1}=y_{n}, \\
y_{n+1}=-b x_{n}+d y_{n}-y_{n}^{3}+\xi_{n},
\end{gathered}
$$

where $\xi_{n}$ is zero-mean white Gaussian noise of variance $D$. Due to symmetry, the noise-free system (1) has pairs of coexisting attractors for $b=0.2$ and $2.0 \leqslant d \leqslant 2.745$, the basins of which are separated by a boundary that may be either smooth or fractal, depending on the chosen values of parameters. We chose for our studies $b=0.2$ and $d=2.7$, which corresponds to there being two coexisting CA basins separated by an LD FBB. One of them is shown in Fig. 1(a). The fractal dimension of the boundary, determined numerically by using the "uncertainty exponent" technique [31], is 1.844 72. The chaotic attractors (CAs) in Eqs. (1) appear as the result of a period-doubling cascade and, for the chosen values of parameters, each of them consists of two disconnected parts. It should be noted that the properties of this map, including the structures both of its CA and of its locally disconnected FBB, are generic for a wide class of maps and flow systems $[4,6]$. For instance, a similar map was introduced recently to model the localized breathing oscillations of Bose-Einstein condensates in periodic traps [32]. Taken together with the results of our investigations of escape in
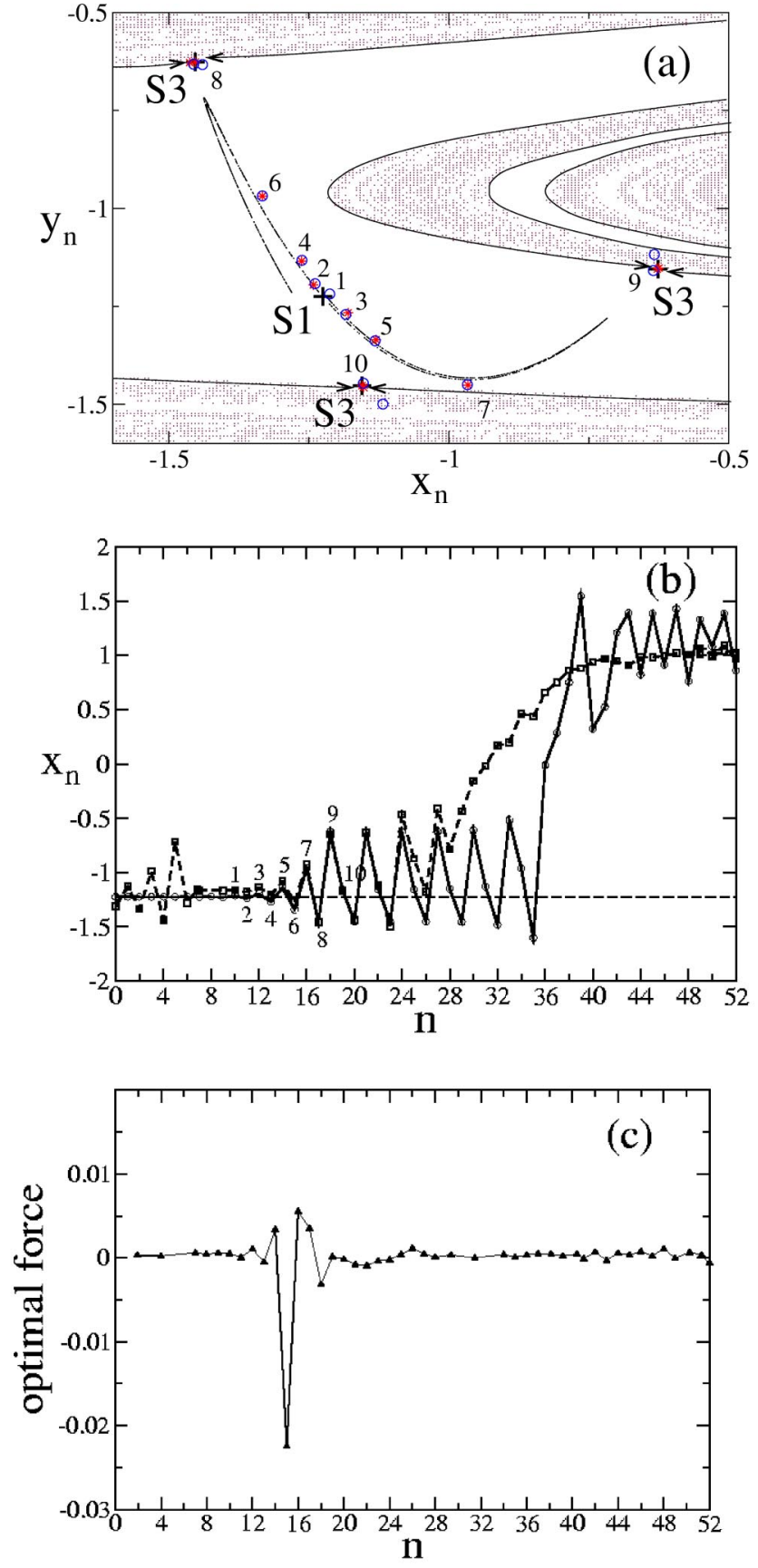

FIG. 1. (a) (Color online) One of the coexisting CAs is indicated by the centrally placed filled curve. Its basin of attraction is shown in white, whereas points belonging to the other basin are shaded in gray. The most probable escape path connecting the CA with the period-3 saddle cycle $S 3$ lying on the fractal boundary is shown by the sequentially numbered small circles (boundary value problem) and stars (Monte Carlo simulations), which practically coincide. The remaining unnumbered circles show how the MPEP evolves, just outside the basin of attraction of the initial state. (b) The $x$ coordinates of the optimal escape paths shown in (a) which were obtained both from the Monte Carlo simulations with $D=10^{-5}$ (dashed line) and from the solution of the boundary value problem (solid line). The abscissa of the saddle point $S 1$ is shown by the thin-dashed line. (c) The optimal fluctuational force moving the system (1) to the LD FBB. All units are dimensionless. 
other systems, these considerations allow us to conclude that the escape mechanism we describe is indeed a typical one.

\section{A. Statistical analysis of escape trajectories}

We excited the system (1) with weak noise and collected both the trajectories that provide escape paths from one CA to the other and the corresponding realizations of noise that induced them. Averaging a few hundred such escape trajectories and noise realizations, we obtained the optimal escape path and the corresponding optimal force, which are shown in Fig. 1. The results of this statistical analysis allow us to determine the boundary conditions near the $\mathrm{CA}$ and on the FBB, and to demonstrate the uniqueness of the MPEP. It can be seen in particular that the system (1) leaves the CA falling into a small neighborhood of the saddle point of period 1 (S1) located between its two disconnected parts. Its stable manifold separates the parts of the CA, while the unstable one approaches the CA. Furthermore, the system makes a few iterations in some small neighborhood of $S 1$ [small plateau in Fig. 1(b)] and then moves in a few steps to the FBB, crossing it at the saddle point of period $3(S 3)$.

Calculations have shown that $S 3$ for the chosen parameter values lies on the FBB. Moreover, its stable manifold (solid black line in Fig. 1) is dense [33] in the FBB and detaches the open neighborhood including an attractor from the FBB itself, thus allowing us to classify it as an accessible boundary point $[23,29,34]$. Indeed, by definition a boundary point $P$ is accessible from a region if there is a curve of finite length connecting $P$ to a point in the interior of the region such that no point of the curve lies in the boundary except for $P$. In the case of the saddle $S 3$, the part of the unstable manifold of $S 3$ approaching the CA and lying inside the open neighborhood plays the role of such a curve. It should be noted that boundary point $S 3$ is the only saddle point belonging to the homoclinic structure whose stable and unstable manifolds are not tangent to each other. All other homoclinic points are buried in the FBB and inaccessible from the open neighborhood including $\mathrm{CA}$ because of such tangencies. Moreover, in the absence of noise, $S 3$ is the only saddle point in the FBB from which the noise-free system (1) can relax to an attractor in a finite number of iterations. For all other initial conditions taken in inaccessible saddle points, the noise-free system (1) must stay inside the FBB infinitely long [29]. In other words, the saddle point $S 3$ is the homoclinic point closest to the CA whose stable manifold separates the interior, including the $\mathrm{CA}$, from the $\mathrm{FBB}$. This situation is similar, in some respects, to the case of fluctuational transitions across the smooth basin boundary formed by the stable manifold of a saddle boundary point. For that case, it is well known that phase trajectories approach a saddle point with zero velocity. For this reason, the action calculated in some small neighborhood of the separatrix takes its minimal value at a saddle point [35]. The present case is much more intricate due to the far more complicated structure of the basin boundary and the large number of saddles embedded in the boundary.

\section{B. Variational formulation of the problem}

Calculation of the MPEP requires an understanding of the mechanism of escape from the initial state to the boundary of its basin of attraction. According to the Hamiltonian theory of fluctuations valid in the limit $D \rightarrow 0$ [13-16], the MPEP is the path which minimizes the "energy" defined as [36]

$$
S=\frac{1}{2} \sum_{n=1}^{N} \vec{\xi}_{n}^{T} \vec{\xi}_{n}
$$

Here the minimization has to be performed over all possible realizations of noise $\left\{\xi_{n}\right\}$ that induce a transition of the system (1) from the CA (with the initial condition on the $S 1$ ) to the FBB. The constraint between coordinates $\left\{x_{n}\right\}$ and $\left\{\xi_{n}\right\}$ defined by Eq. (1) is implemented through introduction of a set of Lagrange undetermined multipliers $\left\{\lambda_{n}\right\}$ [15]. In this way, the minimization of energy (2) over $\left\{\xi_{n}\right\}$ is equivalent to the minimization of

$$
L=\frac{1}{2} \sum_{n=1}^{N} \vec{\xi}_{n}^{T} \vec{\xi}_{n}+\sum_{n=1}^{N} \vec{\lambda}_{n}^{T}\left[\vec{x}_{n+1}-\vec{f}\left(x_{n}\right)-\vec{\xi}_{n}\right],
$$

over $\left\{\xi_{n}\right\},\left\{\lambda_{n}\right\}$, and $\left\{x_{n}\right\}$. Here $\vec{f}(x)$ is the deterministic part of the map.

The result of the minimization is the following areapreserving map:

$$
\begin{gathered}
x_{n+1}=y_{n}, \\
y_{n+1}=-b x_{n}+d y_{n}-y_{n}^{3}+\lambda_{n}^{y}, \\
\lambda_{n+1}^{x}=\left(d-3 x_{n+1}^{2}\right) \lambda_{n}^{x} / b-\lambda_{n}^{y} / b, \\
\lambda_{n+1}^{y}=\lambda_{n}^{x} .
\end{gathered}
$$

The evolution of the "energy" along the solution of Eq. (3) is governed by

$$
S_{n+1}=S_{n}+\frac{1}{2}\left(\lambda_{n}^{y}\right)^{2}
$$

\section{Boundary conditions}

In order to describe the process of escape from the vicinity of the initial structure, a path solution of the extended map is required that minimizes the "energy" (4). We want to describe here the boundary conditions on the basin boundary for such a trajectory and we seek to prove that they have to be chosen on an accessible saddle on the boundary. In what follow, we refer to the notation in Fig. 2: $\Omega 1$ indicates the accessible part of the basin of attraction of the stable structure; $d \Omega$ is the accessible part of the basin boundary, i.e., the stable manifold of the accessible saddle $S 3$; it separates $\Omega 1$ from the remaining part of the coordinate space, here indicated as $\Omega 2 . \Omega 2$ is the set of all points in the space not accessible from $\Omega 1$. We start by proving that $\Omega 1$ is mapped into a subset of $\Omega 1$. This means that no points in $\Omega 1$ are mapped into $\Omega 2$. Composed of accessible points, $\Omega 1$ is a connected set (more precisely an arcwise connected set), while $\Omega 1$ and $\Omega 2$ are separated by $d \Omega$. As the continuous image of a connected set is a connected set, and at least one point of $\Omega 1$ is mapped inside $\Omega 1$, the whole $\Omega 1$ is mapped 
into a subset of itself. In other words, a noise-free trajectory cannot "jump over" the basin boundary. As a second step, we give an argument that proves that an "energy"-optimal escape path out of $\Omega 1$ should terminate on the basin boundary. Let us proceed as follows. The escape is realized when a point $x$ inside $\Omega 1$ is mapped into a point $y^{*}$ in $d \Omega$ or in $\Omega 2$. From the previous argument, we know that $f(x)$ (the noisefree image of $x$ ) is still inside $\Omega 1$. The "energy" cost to be minimized for the transition is $\frac{1}{2}\left[y^{*}-f(x)\right]^{T}\left[y^{*}-f(x)\right]$, on all possible $y^{*} \in\{d \Omega, \Omega 2\}$. The "energy" cost is clearly minimal for a point $y$ on $d \Omega$ (see Fig. 2). Finally, every point on $d \Omega$ can be connected via a noise-free path to the saddle embedded in it. This means that among all possible points on $d \Omega$, the saddle has the least "energy" and that the escape must take place through the accessible saddle $S 3$.

In order to describe the escape process, therefore, the system (3) has to be solved with the following boundary conditions:

$$
\lim _{n \rightarrow-\infty} \lambda_{n}^{y}=0, \quad\left(x_{n}^{0}, y_{n}^{0}\right) \in S 1, \quad\left(x_{n}^{1}, y_{n}^{1}\right) \in S 3 .
$$

The MPEP is the solution of this boundary-value problem that minimizes the cost (4). It is known that, in the phase space, the MPEP is a heteroclinic trajectory connecting $S 1$ and $S 3$ [37-41]. In general, the location of this trajectory is a very complicated problem, due to the singular shape of the unstable manifold of $S 1$ [40-42] and the presence of multiple local minima of the energy [43]. The key step in performing the minimization and solving the boundary-value problem is the description of the family of solutions of Eq. (3) on the unstable manifold of $S 1$ by the use of an appropriate number of parameters [44]. In the vicinity of $S 1$, the unstable manifold is a plane in the phase space. It can be described by introduction of a linear constraint between the coordinates and "momenta" $\vec{\lambda}=M \delta \vec{x}$, where $\delta \vec{x}$ represents the displacement from $S 1 . M$ is a real matrix. In order to calculate the coefficients in the $M$ matrix, the system (3) is expanded about $S 1$ to give

$$
\begin{gathered}
\delta x_{n+1}=A \delta x_{n}+\lambda_{n}, \\
\lambda_{n+1}=A^{-1 T} \lambda_{n},
\end{gathered}
$$

where $A$ is the Jacobian matrix of the original map calculated in $S 1$.

The eigenvalues for the map (6) are $\alpha_{1}, \alpha_{2}, \alpha_{1}^{-1}, \alpha_{2}^{-1}$. The stable eigenvectors associated with the contracting eigenvalues are called $e_{s 1}$ and $e_{s 2}$, where the index $s$ indicates stable. The unstable eigenvectors are denoted by $e_{u 1}$ and $e_{u 2}$. A generic point on the unstable manifold can be written as a combination of $e_{u 1}$ and $e_{u 2}$,

$$
\left(\begin{array}{l}
\vec{x} \\
\vec{\lambda}
\end{array}\right)=c_{u 1}\left(\begin{array}{l}
\vec{e}_{u 1 x} \\
\vec{e}_{u 1 \lambda}
\end{array}\right)+c_{u 2}\left(\begin{array}{l}
\vec{e}_{u 2 x} \\
\vec{e}_{u 2 \lambda}
\end{array}\right) .
$$

Here $\vec{u}_{u 1,2 x}$ and $e_{u 1,2 \lambda}$ represent the $x$ and $\lambda$ components of the eigenvectors $\vec{e}_{u 1,2}$. Writing the vectors $x$ and $\lambda$ explicitly, using their components $x, y, \lambda_{x}, \lambda_{y}$, one obtains a set of linear equations,

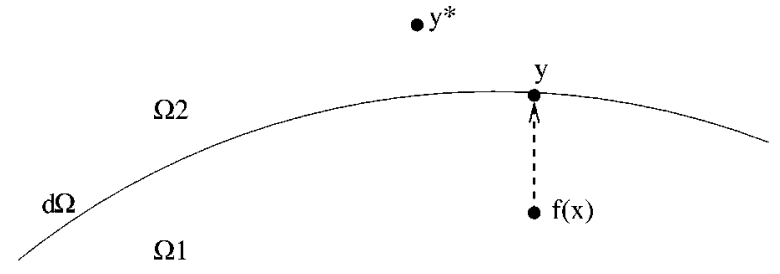

- $\mathrm{x}$

FIG. 2. Details of the escape process: $x$ is a point inside the accessible basin of attraction $\Omega 1, f(x)$ is the noise-free image of $x$, and $y$ is the point on the boundary $d \Omega$ that minimizes the "energy" cost; $y^{*}$ is another (nonoptimal) point in $\Omega 2$ (the set of all points in the space is inaccessible from $\Omega 1$ ). The units are dimensionless.

$$
\begin{aligned}
\left(\begin{array}{c}
x \\
y
\end{array}\right) & =\left(\begin{array}{ll}
e_{u 1 x} & e_{u 2 x} \\
e_{u 1 y} & e_{u 2 y}
\end{array}\right)\left(\begin{array}{l}
c_{u 1} \\
c_{u 2}
\end{array}\right), \\
\left(\begin{array}{c}
\lambda_{x} \\
\lambda_{y}
\end{array}\right) & =\left(\begin{array}{ll}
e_{u 1 \lambda_{x}} & e_{u 2 \lambda_{x}} \\
e_{u 1 \lambda_{y}} & e_{u 2 \lambda_{y}}
\end{array}\right)\left(\begin{array}{c}
c_{u 1} \\
c_{u 2}
\end{array}\right) .
\end{aligned}
$$

Use of standard linear algebra techniques yields a linear relationship between $\vec{x}$ and $\vec{\lambda}$,

$$
M=\left(\begin{array}{ll}
e_{u 1 \lambda_{x}} & e_{u 2 \lambda_{x}} \\
e_{u 1 \lambda_{y}} & e_{u 2 \lambda_{y}}
\end{array}\right)\left(\begin{array}{ll}
e_{u 1 x} & e_{u 2 x} \\
e_{u 1 y} & e_{u 2 y}
\end{array}\right)^{-1} .
$$

Thus a single trajectory in the pattern of solutions can be defined by providing its initial conditions in the coordinate space and the corresponding momenta can be obtained using the matrix $M$. The whole family of trajectories on the unstable manifold can be defined by supplying a region of initial conditions in a neighborhood of $S_{1}$. A possible choice can be, for example, the area included between two circles centered in $S 1$ with radii $r_{1}$ and $r_{2}$ chosen so as to include all possible trajectories in the pattern. The parameters used to describe a single trajectory can be the angular position and the distance $r_{1} \leqslant r<r_{2}$ from $S_{1}$. The evolution of a trajectory is followed until it exits the boundary and the escape "energy" is then recorded. In this way, the "energy" is defined as a real function in the space of parameters. For the system (1), the parameter space is diffeomorph [45] to a torus $T^{2}$, i.e., it can be mapped into a torus $T^{2}$ using an invertible function $h$ such that $h$ and $h^{-1}$ are $C^{\infty}$. The "energy" function can be very wild and singular [44] but a minimization is nevertheless still possible. The MPEP found by this method is shown in Fig. 1. It can be seen that the MPEP predicted by the Hamiltonian theory coincides with that obtained by statistical analysis of the escape trajectories in Monte Carlo simulations. Note that no action is required to bring the system to another attractor after it has reached the accessible orbit on the FBB.

\section{Structure of the escape path inside the FBB}

An analysis of the structure of escape paths inside the FBB has shown that the homoclinic saddle points play a key 
role in its formation. In the system (1), we observe an infinite sequence of saddle-node bifurcations of period 3, 4, 5, 6, $7, \ldots$, at parameter values $d_{3}<d_{4}<d_{5}<d_{6}<d_{7} \cdots$, caused by tangencies of the stable and unstable manifolds of the saddle point $\mathrm{O}$ at the origin. The homoclinic orbits appearing as a result of these bifurcations were classified earlier as original saddles, and it was also shown that their stable and unstable manifolds cross each other in a hierarchical sequence [29]. It is this deterministic structure of the manifolds of the original saddles that determines the fluctuational escape mechanism across the LD FBB. Indeed, to escape from a CA, the system must first cross the stable manifold of the accessible orbit, and then the stable manifolds of the other original saddles, in a predetermined hierarchical sequence. Once the system crosses the stable manifold of a saddle orbit, it relaxes noisefree to the corresponding orbit, which it then leaves along its unstable manifold. Therefore, the hierarchical interrelation between original saddles involved in the escape has to be closely linked to eigenvalues of the Jacobian at these saddles (also known as multipliers of the periodic orbit [46]), characterizing their local stability with respect to motion on the manifolds.

To quantify this interrelation, we introduce a parameter

$$
\mu=\frac{\log \rho_{s t}\left(x_{i}\right)}{\log \rho_{u n}\left(x_{i}\right)},
$$

where $\rho_{s t}\left(x_{i}\right)$ and $\rho_{u n}\left(x_{i}\right)$ are the multipliers of the saddle point $x_{i}$ corresponding to the stable and unstable directions, respectively. This conclusion accords with the fact that the natural measure $\eta$ on a two-dimensional chaotic nonattracting set is concentrated along its unstable manifold and can be represented via unstable eigenvalues of unstable orbits: $\eta(C)=\Sigma 1 / \log \rho_{\text {un }}\left(x_{i}\right)$, where $C$ is the region of phase space containing the chaotic saddle, $\rho_{u n}\left(x_{i}\right)$ is the multiplier corresponding to the unstable manifold, and the summation is over all the unstable orbits $x_{i}$ in $C$ [47] (cf. [48]). Calculations have shown that, for the original saddles of periods 3 , $4,5,6,7,8, \ldots$ in Eq. (1), the following hierarchical sequence of index $\mu$ values occurs: $\mu_{3}=3.339, \mu_{4}=3.080, \mu_{5}=2.999$, $\mu_{6}=2.339, \mu_{7}=1.958$, and $\mu_{8}=1.539$. Moreover, the values of $\mu$ corresponding to the other homoclinic saddle cycles are close to zero. Correspondingly, the probability of finding the system in their neighborhood tends to zero. Our studies of the effect of noise on this deterministic structure proceeded via analysis of relaxational trajectories inside the LD FBB. They have shown that homoclinic tangle is robust to noiseinduced perturbations: the addition of noise caused only a small increase in the probability for the system to escape via saddle cycles of large period, increasing the time of wandering inside the homoclinic tangle [23].

\section{FLUCTUATIONAL TRANSITIONS AND "FRACTAL- FRACTAL" BASIN BOUNDARY CRISIS}

\section{A. Henon map}

To illustrate the above mechanism of transition across the LD FBB, let us consider the situation when the heteroclinic chain shown in Fig. 3 is broken, for instance, between the

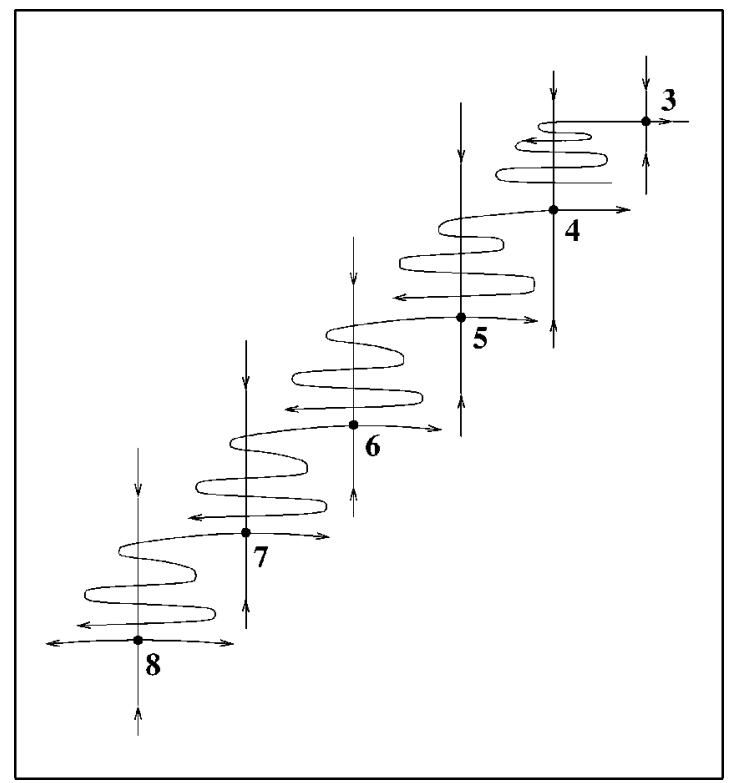

FIG. 3. Illustration of the hierarchical heteroclinic intersections between the stable and unstable manifolds of the original saddles. The units are dimensionless.

saddles of period 3 and 4 . Such a situation leads to the socalled "fractal-fractal" boundary metamorphosis of the LD FBB observed for the first time in the Henon map,

$$
\begin{gathered}
x_{n+1}=A-x_{n}^{2}-J y_{n}+\xi_{n}^{1}, \\
y_{n+1}=x_{n}+\xi_{n}^{2},
\end{gathered}
$$

which we treated as a model excited by two statistically independent Gaussian noise sources whose correlation functions are $\left\langle\xi_{n}^{(1,2)} \xi_{m}^{(1,2)}\right\rangle=2 D \delta_{n m}$. It is well known that the noisefree system (10) is of a generic character, and that it can demonstrate a rich variety of bifurcations and basin transformations. In particular, the "fractal-fractal" basin boundary metamorphosis which manifests itself as a sudden jump of the basin boundary inwards, into the open neighborhood containing the attractor, is observed for certain values of control parameters [29]. In our studies, we fixed parameter $J=0.3$ and varied parameter $A$ within the range $1.38 \leqslant A \leqslant 1.405$. As can be seen from Fig. 4(a), the basin boundary separating the basin of attraction of the stable orbit from the basin of attraction of infinity is fractal for $A=1.38$; its fractal dimension is 1.53. There exists an accessible boundary point of period 4 $(S 4)$, whose stable manifold detaches the open neighborhood from the LD FBB. The unstable manifold of the accessible point $S 4$ has two branches. One of these slices through the basin boundary at a Cantor set of points, while the other lies entirely in the open neighborhood and does not touch the stable manifold of the same accessible boundary point until the crisis. At the value $A \cong 1.396$ where it becomes tangent and then crosses the stable manifold, the saddle point $S 4$ ceases to be the accessible orbit leading to the crisis of the LD FBB and causing the observed sudden jump of the boundary inside the white region [see Fig. 4(b)]. At the same value, the unstable manifold of the saddle point of period 3 

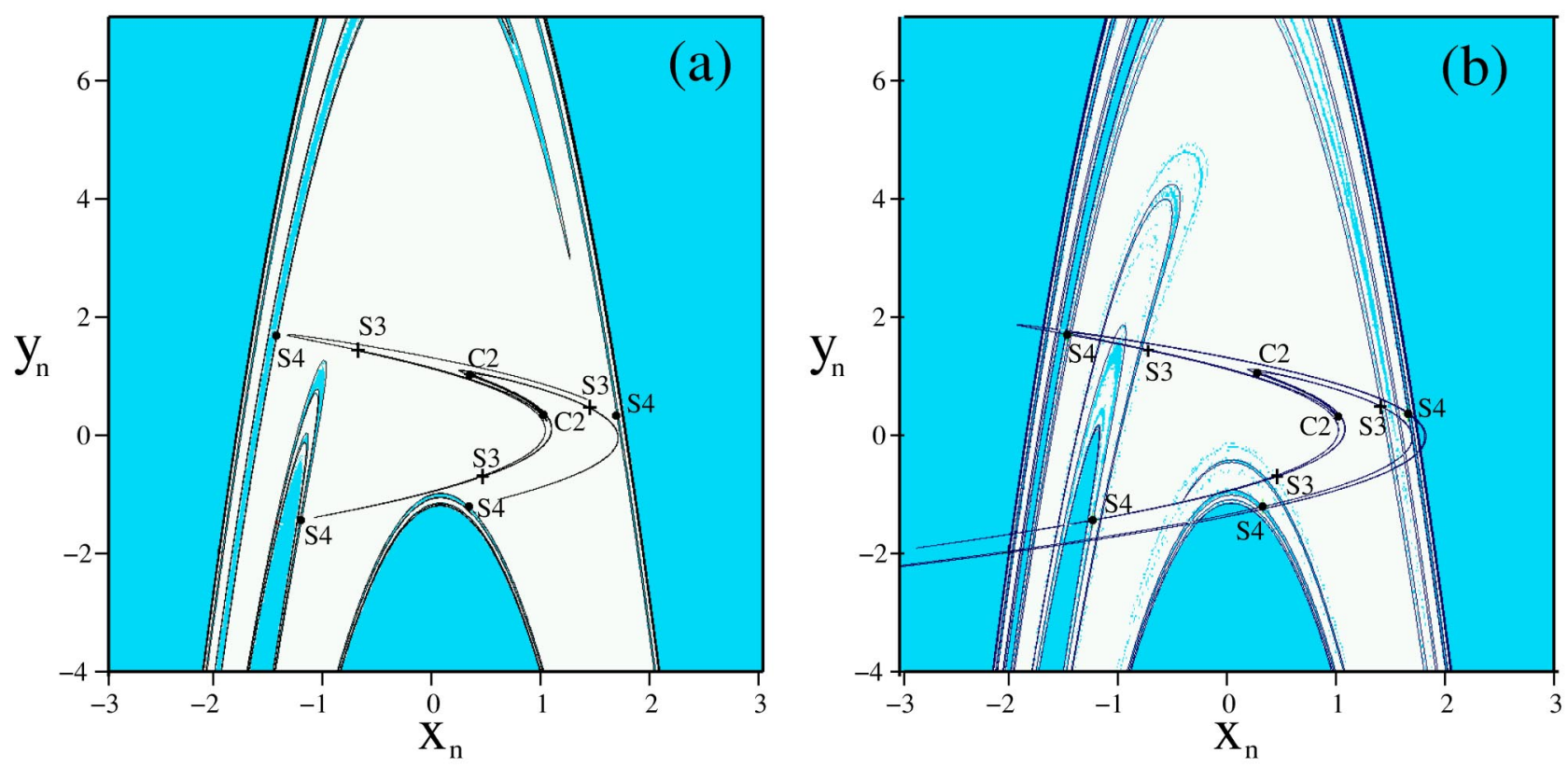

FIG. 4. (Color online) (a) The LD FBB separating the stable point of period 2 (labeled as $C 2$ ) from the attractor at infinity, at $A$ $=1.38$, before the "fractal-fractal" basin boundary metamorphosis (dimensionless units). The unstable manifold of the saddle point of period $3(S 3)$ and the stable manifold of the accessible boundary point of period 4 (S4), indicated by the solid lines, do not intersect. (b) The LD FBB at $A=1.405$ after the "fractal-fractal" boundary crisis. The saddle point $S 3$ has become the accessible boundary point, and $S 4$ lies inside the LD FBB which has jumped inside the open neighborhood containing the stable point $C 2$.

(S3), lying inside the white region, crosses the stable manifold of the saddle $S 4$ and joins the homoclinic structure forming the LD FBB. After the transition, the saddle point $S 3$ becomes an accessible boundary point, whereas the point $S 4$ becomes buried in the basin boundary. The exchange of accessible orbits is the essence of the "fractal-fractal" boundary metamorphosis [29].

Now, let us consider noise-induced escape through the LD FBB in the system (10) for the control parameter value $A$ $=1.38$, preceding the "fractal-fractal" boundary crisis. As we already mentioned above, the saddle point $S 3$ lies inside the basin of attraction of $C 2$ whose unstable manifold also lies entirely inside that basin. To find the optimal escape path, we used the same technique as before, monitoring the system (10) all the time during the iteration process and collecting both the escape trajectories and the corresponding realizations of noise inducing the fluctuational transitions from $C 2$ to infinity. An analysis of the collected escape trajectories shows that escape always occurs via the saddle point $S 3$ and the accessible boundary point $S 4$. Points forming the optimal escape path from $C 2$ to the LD FBB are shown in Fig. 5(a). To understand why escape trajectories always pass through S3, which does not belong to the boundary, we calculated its stable manifold which, as seen from Fig. 5(a), lies entirely inside the basin of $C 2$ and is positioned closer to the attractor than the LD FBB. For this reason, the only way for the system (10) to reach LD FBB is to cross, first, the stable manifold of the saddle $S 3$ and then, second, move to the LD FBB crossing it at accessible boundary point $S 4$.

To find the MPEP in the limit of $D \rightarrow 0$, we repeated the procedure described in Sec. II for the following enlarged system:

$$
\begin{gathered}
x_{n+1}=A-x_{n}^{2}-J y_{n}+\lambda_{n}^{x}, \\
y_{n+1}=x_{n}+\lambda_{n}^{y}, \\
\lambda_{n+1}^{x}=\lambda_{n}^{y}, \\
\lambda_{n+1}^{y}=-\left(\lambda_{n}^{x}+2 x_{n} \lambda_{n}^{y}\right) / J,
\end{gathered}
$$

considering as boundary conditions the stable point $C 2$ and the accessible boundary point $S 4$. The results of our calculations are presented in Fig. 5(a). The MPEP starting from $C 2$ reaches, first, the saddle point $S 3$ and then goes to the $\mathrm{LD}$ FBB, crossing it at the point $S 4$. Further relaxation does not require any external force and is governed by the homoclinic tangle. Its hierarchical structure is characterized by the following values of the parameter $\mu: \mu_{3}=3.5076, \mu_{4}=2.7699$, $\mu_{5}=2.5396$, and $\mu_{6}=2.3898$. It is clearly seen from Fig. 5 that the MPEP coincides with the optimal escape path computed from the Monte Carlo simulations. Thus, there are two heteroclinic trajectories in the phase space of the system (12) connecting points $C 2$ and $S 4$. The first one connects the saddle points $C 2$ and $S 3$, while the second one connects $S 3$ and $S 4$. Thus it seems that, in the presence of noise, the saddle point $S 3$ starts to play the role of an accessible boundary point instead of $S 4$.

We also calculated the optimal fluctuational force moving our system to the LD FBB for two different values of the control parameter $A$ corresponding to the states before and after the "fractal-fractal" boundary crisis. The results of our calculations are presented in Fig. 5(b). It can be seen that, before the boundary crisis, the system (10) still needs to be 

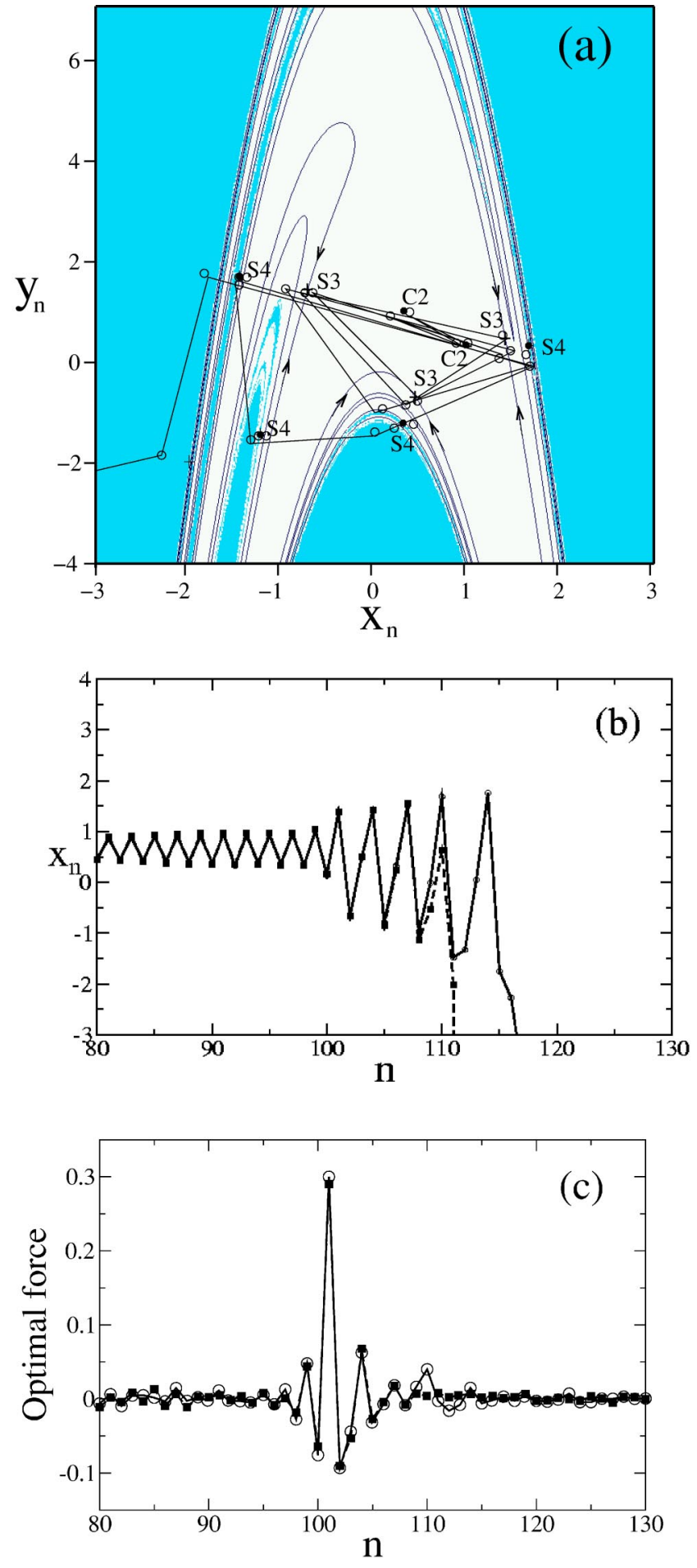

FIG. 5. (a) (Color online) The LD FBB of the system (10) before the boundary crisis $A=1.38$. The stable manifold of the saddle point $S 3$ (indicated by crosses) shown by the solid lines. The optimal escape path obtained from the Monte Carlo simulations with $D=1.3 \times 10^{-3}$ is shown by the thick solid line, while points belonging to the MPEP are shown by open circles. (b) The $x$ coordinates of the optimal escape paths before (solid line) and after (dashed line) the "fractal-fractal" boundary crisis at $A=1.405$. (c) The optimal fluctuational forces calculated before (black filled squares) and after (open circles) the crisis. All units are dimensionless.
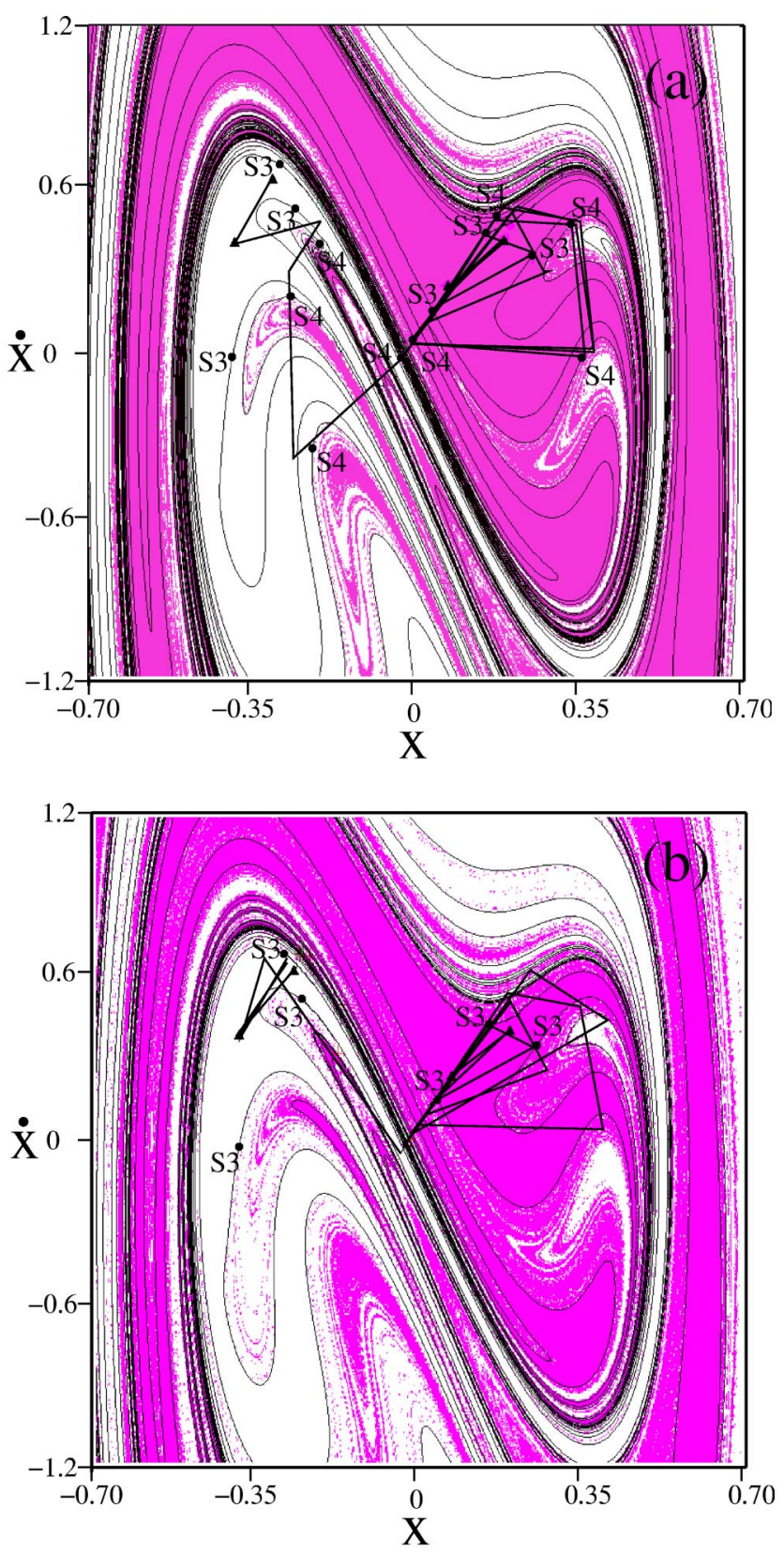

FIG. 6. (Color online) (a) Stroboscopic $(\Omega t$ $=0,2 \pi / \Omega, 4 \pi / \Omega, \ldots)$ section of the system (12) before the "fractal-fractal" basin boundary crisis at $\Gamma=1, \alpha=10, \beta=100, \Omega$ $=3.76$, and $A=0.9$ (dimensionless units). Two coexisting stable points of period 2 are indicated by filled triangles. Their basins of attraction are colored in white and gray, respectively. The stable manifolds of the saddle points $S 3$ and $S 4$ (indicated by filled circles) are shown by the solid thin lines. The optimal escape path obtained from the Monte Carlo simulations with $D=3 \times 10^{-5}$ is shown by the thick solid line. (b) The same stroboscopic section after the boundary crisis at $A=0.915$. All other parameters have the same values as in the previous figure. The optimal escape path obtained from the simulations with $D=2 \times 10^{-5}$ is shown by the thick solid line.

excited to move from the saddle $S 3$ to the boundary point $S 4$, whereas after the crisis it does not. Apparently, this fact is caused by the absence of any deterministic structure able to sustain the transition before the boundary crisis. 


\section{B. Duffing oscillator}

A similar situation is observed for the periodically driven Duffing oscillator excited by white Gaussian noise,

$$
\ddot{x}+\Gamma \dot{x}-\alpha x+\beta x^{3}=A \cos \Omega t+\sqrt{2 D} \xi(t),
$$

where $\Gamma$ is the coefficient of dissipation, $\alpha$ and $\beta$ are parameters defining the shape of a potential, $A$ and $\Omega$ are the amplitude and frequency of external force, and $D$ is the intensity of the noise $\xi(t)$. It is known that for some values of control parameters, the noise-free system (12) can demonstrate the same type of boundary crisis as the Henon map considered above. Using the same technique as before, we calculated the optimal escape paths for the two different values of the driving amplitude corresponding to the states before and after the "fractal-fractal" boundary crisis. The results of our calculations are pictured in Fig. 6. As is clearly seen from this figure, the system (12) moves to the FBB via saddle points $S 3$ and $S 4$ before the crisis, while after that it jumps via point $S 3$ only. The close correspondence between results obtained for the two different kinds of systems allows us to conclude that, under the influence of noise, the saddle point $S 3$ acquires the features of an accessible boundary point. However, this saddle point does not belong to the FBB. This fact does not allow us to claim that weak noise can induce a "fractal-fractal" boundary crisis. However, taking into account the significant influence of $S 3$ on the escape process, we may speak about a noisy precursor of the crisis on the LD FBB, which manifests itself in observed structure of the optimal escape path.

We remind the reader that the notion of a noisy precursor was originally introduced by Wiesenfield [49] during studies of noisy dynamical systems near bifurcations. It is well known that dynamical systems are very sensitive to fluctuations near a bifurcation point. It was shown that even weak noise is able to induce the appearance of additional peaks in a power spectrum of response of a noisy dynamical system indicating its proximity to the bifurcation point. It is also known that the response of a dynamical system near bifurcation can be characterized by a bell-shaped dependence of the signal-to-noise ratio on the noise intensity. It is this fact that allows one to speak of coherence resonance at the noisy precursor of a bifurcation [50]. However, all previous studies were concerned with the different bifurcations of attractors themselves, whereas in many real situations dynamical instabilities can also be caused by bifurcations of the basins of attraction [51]. Our above results clearly show that analysis of the structure of the optimal escape paths allows us to draw a conclusion about the presence of an instability caused by bifurcation of the basin boundary.

\section{FLUCTUATIONAL TRANSITIONS ACROSS AN LC FBB}

We now consider the same escape problem, but in an system possessing a LC FBB. This type of FBB is generally observed in two-dimensional noninvertible analytic and nonanalytic maps [4,52]. We will take as our model a typical nonanalytic quadratic map driven by noise,
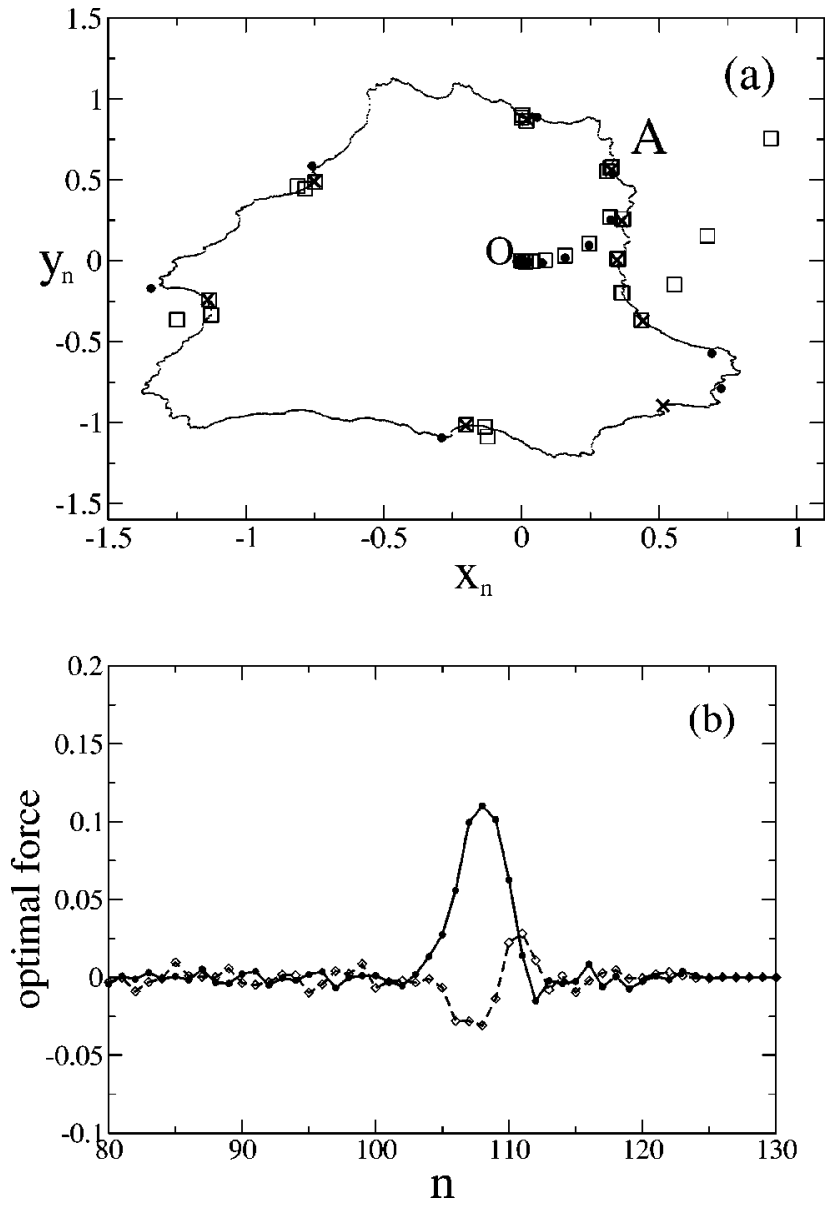

FIG. 7. (a) The locally connected FBB (solid closed curve), unstable node of period 9 (crosses), and points on the optimal escape path obtained from the Monte Carlo simulations (filled circles) with $D=5 \times 10^{-3}$. Points of the MPEP are shown by empty squares. (b) The $x$ (solid line) and $y$ (dashed line) components of the optimal fluctuational force. All units are dimensionless.

$$
\begin{gathered}
x_{n+1}=x_{n}^{2}-y_{n}^{2}+\alpha x_{n}+\xi_{n}^{1}, \\
y_{n+1}=2 x_{n} y_{n}+\alpha x_{n}+\beta y_{n}+\xi_{n}^{2},
\end{gathered}
$$

where $\xi_{n}^{1}, \xi_{n}^{2}$ are statistically independent sources of white, Gaussian noise of zero mean that are of equal intensity $D$. For the chosen values of the control parameters $\alpha=0.7, \beta$ $=0.5$, this map has stable points at the origin and at infinity, separated by the LC FBB. The boundary contains an infinite set of repelling points and no saddle points. It is the Julia set homeomorphic to a circle. To find the boundary condition on the LC FBB and the optimal escape path, we use exactly the same technique as in the case of the LD FBB, above. The results of our calculations are presented in Fig. 7. As can clearly be seen from this figure, the system (13) leaves the stable point $O$ at the origin along a unique optimal escape path and approaches the LC FBB at the unique point shown in Fig. 7(a). Moreover, our calculations have shown that the optimal fluctuational force [see Fig. 7(b)] becomes equal to zero at this moment. 
According to our previous results, this means that the system (13) reached the boundary at this point, and its further relaxation to infinity is noise-free and completely specified by the deterministic structure of the FBB. Our calculations have shown that the boundary point $A$ corresponds exactly to the repelling boundary point of period 9 , which plays the role of the unique boundary condition on this LC FBB. Moreover, our studies have shown that this repelling point plays the role of the boundary condition over a wide range of the control parameters $\alpha$ and $\beta$. Note that noise-induced escape from the attractor surrounded by an LC FBB in Eq. (13) was considered earlier in the pioneering work of Grassberger [15], who succeeded in calculating the optimal escape path, albeit without finding the boundary condition on the LC FBB or the mechanism of escape.

To find the MPEP, we treated the following enlarged system:

$$
\begin{gathered}
x_{n+1}=x_{n}^{2}-y_{n}^{2}+\alpha x_{n}+\lambda_{n}^{x}, \\
y_{n+1}=2 x_{n} y_{n}+\alpha x_{n}+\beta y_{n}+\lambda_{n}^{y}, \\
\lambda_{n+1}^{x}=\left[\left(2 x_{n}+\beta\right) \lambda_{n}^{x}+2 y_{n} \lambda_{n}\right] / A, \\
\lambda_{n+1}^{y}=\left[-\left(2 y_{n}+\alpha\right) \lambda_{n}^{x}+\left(2 x_{n}+\alpha\right) \lambda_{n}^{y}\right] / A,
\end{gathered}
$$

supplemented by the boundary conditions at the steady point $O$ and at the repeller of period 9. Here $A=\left(2 x_{n}+\alpha\right)\left(2 x_{n}\right.$ $+\beta)+2 y_{n}\left(2 y_{n}+\alpha\right)$ is the determinant of the Jacobian written for the system (13). As is seen from Fig. 7(a), the MPEP coincides almost perfectly with the optimal path obtained from the Monte Carlo simulations.

\section{CONCLUSIONS}

We have studied fluctuational transitions between coexisting regular attractors separated by both LD and LC FBBs. We have shown that an accessible point on the FBB plays the role of a unique boundary condition for both types of FBB.
Our statistical analyses of fluctuational trajectories have yielded solutions of the boundary-value problem for both types of $\mathrm{FBB}$, and have revealed the optimal fluctuational forces moving the systems (1) and (13) from one attractor to the other. We were also able to find the unique optimal escape path in both cases. The original saddles forming the homoclinic structure of the system (1) play a key role in the formation of the escape paths inside the LD FBB, and the difference in their local stability defines the hierarchical relationship between them. We have also considered fluctuational transitions in a dynamical system exhibiting a "fractalfractal" boundary crisis. It is shown that noise can effectively move the accessible boundary point inside the domain of attraction, giving rise to a noisy precursor of the boundary crisis that manifests itself in the structure of the optimal escape path. We have generalized the notion of a noisy precursor to the case of a bifurcation of the basin boundary. The results obtained can be applied directly to the other maps and flows having the same type of FBB.

We emphasize that similar behavior is expected for fluctuational transitions between chaotic attractors separated by FBBs [23]. It has therefore become possible to predict a scenario of escape through a fractal boundary using a deterministic analysis of the FBB structure. Furthermore, recent results [22] have shown that the boundary conditions at chaotic attractors can also be related to the homoclinic structure, and thus found deterministically. Among a variety of possible applications, we would mention a new method of chaos control in the presence of fluctuations that can now be developed [53].

\section{ACKNOWLEDGMENTS}

It is a pleasure to acknowledge fruitful discussions with Mark Dykman, Ulrike Feudel, Igor Khovanov, Suso Kraut, Edson Leonel, and Stanislav Soskin. The work was supported by the Engineering and Physical Sciences Research Council (UK), the Russian Foundation for Fundamental Research, and INTAS.
[1] R. Roy, R. Short, J. Durnin, and L. Mandel, Phys. Rev. Lett. 45, 1486 (1980); M. B. Willemsen, M. U. F. Khalid, M. P. van Exter, and J. P. Woerdman, ibid. 82, 4815 (1999); J. Hales, A. Zhukov, R. Roy, and M. I. Dykman, ibid. 85, 78 (2000).

[2] A. M. Yacomotti et al., Phys. Rev. Lett. 83, 292 (1999).

[3] E. Ott, Chaos in Dynamical Systems (Cambridge University Press, Cambridge, UK, 2002).

[4] S. W. McDonald, C. Grebogi, E. Ott, and J. A. Yorke, Physica D 17, 125 (1985).

[5] J. C. Sommerer and E. Ott, Nature (London) 365, 135 (1993); H. E. Nusse and J. A. Yorke, Science 271, 1376 (1996); B. R. Hunt, E. Ott, and E. Rosa, Phys. Rev. Lett. 82, 3597 (1999).

[6] M. L. Cartwright and J. E. Littlewood, Ann. Math. 54, 1 (1951); F. C. Moon and G.-X. Li, Phys. Rev. Lett. 55, 1439 (1985).

[7] P. Reimann, Phys. Rev. E 49, 4938 (1994).
[8] P. Reimann, R. Müller, and P. Talkner, Phys. Rev. E 49, 3670 (1994).

[9] P. Reimann and P. Talkner, Phys. Rev. E 51, 4105 (1995).

[10] M. I. Freidlin and A. D. Wentzel, Random Perturbations in Dynamical Systems (Springer, New York, 1984).

[11] Y. Kifer, Trans. Am. Math. Soc. 321, 505 (1990).

[12] Y. Kifer, Ann. Prob. 18, 1676 (1990).

[13] R. L. Kautz, Phys. Lett. A 125, 315 (1987)

[14] P. D. Beale, Phys. Rev. A 40, 3998 (1989).

[15] P. Grassberger, J. Phys. A 22, 3283 (1989).

[16] R. Graham, A. Hamm, and T. Tel, Phys. Rev. Lett. 66, 3089 (1991).

[17] M. I. Dykman, P. V. E. McClintock, V. N. Smelyanski, N. D. Stein, and N. G. Stocks, Phys. Rev. Lett. 68, 2718 (1992).

[18] D. G. Luchinsky, J. Phys. A 30, L577 (1997); D. G. Luchinsky and P. V. E. McClintock, Nature (London) 389, 463 (1997). 
[19] D. G. Luchinsky and I. A. Khovanov, JETP Lett. 69, 825 (1999).

[20] M. I. Dykman et al., Phys. Rev. A 45, R7678 (1992).

[21] I. A. Khovanov, D. G. Luchinsky, P. V. E. McClintock, and R. Mannella, Phys. Rev. Lett. 85, 2100 (2000).

[22] S. Kraut and C. Grebogi, Phys. Rev. Lett. 92, 234101 (2004).

[23] A. N. Silchenko, S. Beri, D. G. Luchinsky, and P. V. E. McClintock, Phys. Rev. Lett. 91, 174104 (2003).

[24] S. M. Soskin, R. Mannella, M. Arrayás, and A. N. Silchenko, Phys. Rev. E 63, 051111 (2001).

[25] S. M. Soskin, R. Mannella, and P. V. E. McClintock, Phys. Rep. 373, 247 (2003).

[26] I. A. Khovanov, D. G. Luchinsky, R. Mannella, and P. V. E. McClintock, Phys. Rev. Lett. 85, 2100 (2001).

[27] A. L. Fradkov and A. Y. Pogromsky, Introduction to Control of Oscillations and Chaos, Series on Nonlinear Science A (World Scientific, Singapore, 1998), Vol. 35.

[28] S. Boccaletti, C. Grebogi, Y. C. Lai, H. Mancini, and D. Maza, Phys. Rep. 39, 103 (2000).

[29] C. Grebogi, E. Ott, and J. A. Yorke, Physica D 24, 243 (1987).

[30] P. Holmes, Philos. Trans. R. Soc. London, Ser. A 292, 419 (1979).

[31] C. Grebogi, S. W. McDonald, E. Ott, and J. A. Yorke, Phys. Lett. 99A, 415 (1983).

[32] R. Carretero-González and K. Promislow, Phys. Rev. A 66, 033610 (2002).

[33] By "dense" we mean that, for any given point $x$ in the FBB, and arbitrarily small $\epsilon$, a point $y$ on the stable manifold of S3 exists such that $|x-y|<\epsilon$.

[34] A. N. Silchenko, D. G. Luchinsky, and P. V. E. McClintock, Physica A 327, 371 (2003).

[35] M. I. Dykman and M. A. Krivoglaz, Sov. Phys. JETP 50, 30 (1979).

[36] R. Graham, Z. Phys. B 26, 281 (1977).

[37] D. Ludwig, SIAM Rev. 17, 605 (1975).

[38] R. S. Maier and D. L. Stein, J. Stat. Phys. 83, 291 (1996).

[39] R. S. Maier and D. L. Stein, Phys. Rev. Lett. 71, 1783 (1993).
[40] M. I. Dykman, M. M. Millonas, and V. N. Smelyanskiy, Phys. Lett. A 195, 53 (1994).

[41] V. N. Smelyanskiy and M. I. Dykman, Phys. Rev. E 55, 2516 (1997).

[42] R. Graham and T. Tel, Phys. Rev. Lett. 52, 9 (1984).

[43] D. G. Luchinsky, S. Beri, R. Mannella, P. V. E. McClintock, and I. A. Khovanov, Int. J. Bifurcation Chaos Appl. Sci. Eng. 12, 583 (2002).

[44] S. Beri, D. G. Luchinsky, A. Silchenko, and P. V. E. McClintock, Solution of the Boundary Value Problem for Optimal Escape in Continuous Stochastic Systems and Maps in Noise in Complex Systems and Stochastic Dynamics (SPIE, Bellingham, WA, 2003), Vol. 5114, pp. 372-382.

[45] E. A. Jackson, Perspectives of Nonlinear Dynamics (Cambridge University Press, Cambridge, UK, 1989).

[46] L. P. Shilnikov, A. L. Shilnikov, D. V. Turaev, and L. O. Chua, Methods of Qualitative Theory in Nonlinear Dynamics, Series on Nonlinear Science A (Singapore, World Scientific, 1998), Vol. 4.

[47] M. Dhamala and Y. C. Lai, Int. J. Bifurcation Chaos Appl. Sci. Eng. 12, 2991 (2002).

[48] C. Grebogi, E. Ott, and J. A. Yorke, Phys. Rev. A 37, 1711 (1988).

[49] K. Wiesenfeld, J. Stat. Phys. 38, 1071 (1985).

[50] A. Neiman, P. I. Saparin, and L. Stone, Phys. Rev. E 56, 270 (1997).

[51] M. S. Soliman and J. M. T. Thompson, Phys. Rev. A 45, 3425 (1992); J. M. T. Thompson, H. B. Stewart, and Y. Ueda, Phys. Rev. E 49, 1019 (1994); K. J. Spyrou and J. M. T. Thompson, Philos. Trans. R. Soc. London, Ser. A 358, 1735 (2000); M. S. Soliman and J. M. T. Thompson, Dyn. Stab. Syst. 4, 281 (1990)

[52] L. Gardini, C. Mira, J. Barugola, and J. C. Cathala, Chaotic Dynamics in Two-Dimensional Noninvertible Maps (World Scientific, Singapore, 1996).

[53] I. A. Khovanov, N. A. Khovanova, and P. V. E. McClintock, Phys. Rev. E 67, 051102 (2003). 